\title{
TATE CONJECTURE FOR SOME ABELIAN SURFACES OVER TOTALLY REAL OR CM NUMBER FIELDS
}

\author{
CRISTIAN VIRDOL
}

\begin{abstract}
In this paper we prove Tate conjecture for abelian surfaces of the type $\operatorname{Res}_{K / F} E$ where $E$ is an elliptic curve defined over a totally real or CM number field $K$, and $F$ is a subfield of $K$ such that $[K: F]=2$.
\end{abstract}

Keywords: Tate conjecture, abelian surfaces, totally real fields.

\section{Introduction}

Let $X$ be a smooth projective variety defined over a number field $F$. For a prime number $l$, let $H_{e t}^{i}\left(X, \overline{\mathbb{Q}}_{l}\right)$ be the $l$-adic cohomology of $\bar{X}=X \times_{F} \overline{\mathbb{Q}}$. For $K$ a number field, we denote $\Gamma_{K}:=\operatorname{Gal}(\overline{\mathbb{Q}} / K)$. The Galois group $\Gamma_{F}$ acts on $H_{e t}^{i}\left(X, \overline{\mathbb{Q}}_{l}\right)$ by a representation $\rho_{i, l}$. For a finite extension $E$ of $F$, the elements of $V^{i}(X, E):=$ $\left(H_{e t}^{2 i}\left(X, \overline{\mathbb{Q}}_{l}\right)(i)\right)^{\Gamma_{E}}$ are called Tate classes defined over $E$ (here $H_{e t}^{2 i}\left(X, \overline{\mathbb{Q}}_{l}\right)(i)$ is the Tate twist).

Let $U^{i}(X)$ be the $\mathbb{Q}$-linear space of the algebraic subvarieties of $X$ of codimension $i$. We have the $l$-adic cycle map

$$
d_{i, l}: U^{i}(X) \otimes \overline{\mathbb{Q}}_{l} \rightarrow H_{e t}^{2 i}\left(X, \overline{\mathbb{Q}}_{l}\right)(i)
$$

The cohomology classes in the image of this map are said to be algebraic.

For each finite extension $E$ of $F$, we denote by $U^{i}(X, E)$ the subspace of $d_{i, l}\left(U^{i}(X) \otimes \overline{\mathbb{Q}}_{l}\right)$ left fixed by $\Gamma_{E}$. The first part of the Tate conjecture [TA] states that

$$
U^{i}(X, E)=V^{i}(X, E)
$$

i.e. each Tate class is algebraic.

The $L$-function $L^{i}\left(s, X_{/ F}\right)$ attached to the representation $\rho_{i, l}$ converges for $\operatorname{Re}(s)>1+i / 2$. The second part of the Tate conjecture [TA] states that for each

2010 Mathematics Subject Classification: primary: 11F41; secondary: 11F $80,11 \mathrm{R} 42$, $11 \mathrm{R} 80$ 
finite extension $E$ of $F$, the $L$-function $L^{2 i}\left(s, X_{/ E}\right)$ has a meromorphic continuation to the entire complex plane and the order of the pole at $s=i+1$ is equal to

$$
\operatorname{dim}_{\overline{\mathbb{Q}}_{l}} U^{i}(X, E) .
$$

In this paper we consider $K / F$ a quadratic extension of $\mathrm{CM}$ number fields (throughout this paper, in order to simplify the notations, by a CM number field we understand a totally real or a totally complex quadratic extension of a totally real number field). Let $E$ be an elliptic curve defined over $K$, and let $A:=\operatorname{Res}_{K / F} E$ be the abelian surface defined over $F$ obtained from $E$ by restriction of scalars from $K$ to $F$. In this article we prove the first part of the Tate conjecture for the abelian surfaces $A$ (see $\S 4.1$ below). We also prove the second part of the Tate conjecture for the abelian surface $A$ regarded over arbitrary CM finite extensions $F^{\prime}$ of $F$ (see Theorem 4.2 and $\S 4.1$ and $\S 4.2$ below).

\section{Some abelian surfaces}

Let $K / F$ be a quadratic extension of $\mathrm{CM}$ number fields, and let $E$ be an elliptic curve defined over $K$. Consider the surface $A:=\operatorname{Res}_{K / F} E$ defined over $F$.

For a rational prime $l$, let

$$
T_{l}(E)=\lim _{\longleftarrow} E\left[l^{n}\right],
$$

and $V_{l}(E)=T_{l}(E) \otimes \mathbb{Q}$. The Galois group $\Gamma_{K}$ acts on

$$
T_{l}(E) \simeq \mathbb{Z}_{l}^{2}
$$

where $\mathbb{Z}_{l}$ is the $l$-adic completion of $\mathbb{Z}$ at $l$, and also on $V_{l}(E) \simeq \mathbb{Q}_{l}^{2}$, and we obtain a representation

$$
\rho_{E}:=\rho_{E, l}: \Gamma_{K} \rightarrow \operatorname{Aut}\left(T_{l}(E)\right) \simeq \mathrm{GL}_{2}\left(\mathbb{Z}_{l}\right) \subset \operatorname{Aut}\left(V_{l}(E)\right) \simeq \mathrm{GL}_{2}\left(\mathbb{Q}_{l}\right) .
$$

Also consider

$$
T_{l}(A)=\lim _{\longleftarrow} A\left[l^{n}\right],
$$

and $V_{l}(A)=T_{l}(A) \otimes \mathbb{Q}$. The Galois group $\Gamma_{F}$ acts on

$$
T_{l}(A) \simeq \mathbb{Z}_{l}^{4}
$$

and also on $V_{l}(A) \simeq \mathbb{Q}_{l}^{4}$, and we obtain a representation

$$
\rho_{A}:=\rho_{A, l}: \Gamma_{F} \rightarrow \operatorname{Aut}\left(T_{l}(A)\right) \simeq \operatorname{GL}_{4}\left(\mathbb{Z}_{l}\right) \subset \operatorname{Aut}\left(V_{l}(A)\right) \simeq \operatorname{GL}_{4}\left(\mathbb{Q}_{l}\right) .
$$

Then

$$
\rho_{A}=\operatorname{Ind}_{\Gamma_{K}}^{\Gamma_{F}} \rho_{E} .
$$

(We remark that our $l$-adic representations $\rho_{E}, \rho_{A}$, etc. could be regarded as complex representations by using some isomorphism $\overline{\mathbb{Q}}_{l} \stackrel{\sim}{\rightarrow} \mathbb{C}$; we will do this throughout this paper without mentioning it). 
We know that (see Theorem 12.1 of $[\mathrm{M}]$ )

$$
H_{e t}^{1}\left(A, \mathbb{Z}_{l}\right) \simeq T_{l}^{\vee}:=\operatorname{Hom}_{\mathbb{Z}_{l}}\left(T_{l}(A), \mathbb{Z}_{l}\right)
$$

and

$$
\wedge^{r} H_{e t}^{1}\left(A, \mathbb{Z}_{l}\right) \simeq H_{e t}^{r}\left(A, \mathbb{Z}_{l}\right), \forall r
$$

So $H_{e t}^{r}\left(A, \mathbb{Z}_{l}\right)$ is a free $\mathbb{Z}_{l}$-module of $\operatorname{rank}\left(\begin{array}{l}4 \\ r\end{array}\right)$ for any $0 \leqslant r \leqslant 4$.

Since

$$
\rho_{E} \simeq \rho_{E}^{\vee}
$$

we obtain that the action of $\Gamma_{F}$ on $H_{e t}^{r}\left(A, \mathbb{Z}_{l}\right)$ is given by

$$
\wedge^{r} \rho_{A} \simeq \wedge^{r} \operatorname{Ind}_{\Gamma_{K}}^{\Gamma_{F}} \rho_{E}
$$

\section{Known results}

We say that a representation $\rho$ of a group $G$ is dihedral if there exists a normal subgroup $N$ of index 2 in $G$ and a character $\chi: N \rightarrow \mathbb{C}^{\times}$such that $\rho=\operatorname{Ind}_{N}^{G} \chi$. We say that an elliptic curve $E$ defined over some number field $K$ has CM if $\rho_{E}$ is dihedral.

We say that an automorphic representation $\pi$ of $\mathrm{GL}(2) / L$ for some number field $L$ is of CM type if there exists some quadratic Galois character $\eta: I_{L} / L^{\times} \rightarrow \mathbb{C}^{\times}$, with $\eta \neq 1$ such that $\pi \cong \pi \otimes \eta$. If $\pi$ is an automorphic representation of weight 2 of GL(2)/L, then $\pi$ is of CM type if and only if the $l$-adic (complex) representation $\rho_{\pi}$ associated to $\pi$ is dihedral.

It is known that (see for example Proposition 4.5.4 of [HLR]; one has a similar statement for $l$-adic representations associated to elliptic curves over number fields, or for $F$ a CM number field if $\rho_{\pi}$ is known to exist):

Lemma 3.1. If $\pi$ is an automorphic representation of weight 2 of $G L(2) / F$, where $F$ is a totally real field, then one of the following two statements holds:

(i) $\left.\rho_{\pi}\right|_{\Gamma_{L}}$ is irreducible for each finite extension $L / F$.

(ii) There exists a quadratic extension $L / F$ and an algebraic Hecke character $\psi$ of $L$ such that $\rho_{\pi} \cong \operatorname{Ind}_{\Gamma_{\mathrm{L}}}^{\Gamma_{\mathrm{F}}} \psi$.

We know the following result (Theorem 2.1 of $[\mathrm{MP}]$ ):

Lemma 3.2. The tensor product of two 2-dimensional irreducible complex representations of a group is reducible only if either both representations are dihedral or they are the twist of each other by a character.

We know (Proposition 4.1 of $[\mathrm{MP}]$ ):

Lemma 3.3. Suppose that $\pi$ is a cuspidal, non-CM automorphic representation of $G L(2) / K$ for some finite extension $K / \mathbb{Q}$. Suppose that $K$ is a quadratic extension of $k$ and $\tau$ is the automorphism of $K$ over $k$. If $\pi^{\tau} \cong \pi \otimes \chi$ for a Hecke character $\chi$ of $K$, then $\chi$ is trivial when restricted to the idéles of $k$. 
We know (Corrolary 2.6 of $[\mathrm{MP}])$ :

Lemma 3.4. Let $\rho$ be a 2-dimensional irreducible representation of a group $G$. Then $\operatorname{Sym}^{2}(\rho)$ is reducible if and only if $\rho$ is dihedral.

We know (see Lemma 2 of $[\mathrm{K}])$ :

Lemma 3.5. Let $\sigma$ and $\tau$ two $n$-dimensional representations of a group $G$ over $\overline{\mathbb{Q}}_{l}$ and assume that $H$ is an open normal subgroup of $G$ and $\left.\tau\right|_{H}$ is irreducible. Then $\left.\left.\sigma\right|_{H} \cong \tau\right|_{H}$ iff $\sigma \cong \tau \otimes \varphi$ for some $\varphi: G \rightarrow \overline{\mathbb{Q}}_{l}^{\times}$, which is trivial on $H$.

We know (see the main theorem of $[\mathrm{GJ}]$ ):

Lemma 3.6. Let $\pi$ be a cuspidal, non-CM automorphic representations of weight 2 of $G L(2) / K$ for some finite extension $K / \mathbb{Q}$. Then $S y m^{2} \pi$ is a cuspidal automorphic representation of $G L(3) / K$.

\section{Tate Conjecture for abelian surfaces}

In this section we prove the first and second parts of the Tate conjecture for $A$.

\subsection{Algebraicity of the Tate classes}

We have that $A_{/ K} \simeq E \times E^{\tau}$, where $\tau$ is the nontrivial automorphism of $K / F$. Since the first part of the Tate conjecture for $E \times E^{\tau}$ is true (for the details of a proof see the last paragraph of [R1]), and the first part of the Tate conjecture is invariant under extension of the field of definition of the variety, we obtain that the first part of the Tate conjecture for $A$ is true.

\subsection{Some representations}

Since $\left(\begin{array}{l}4 \\ 0\end{array}\right)=\left(\begin{array}{l}4 \\ 4\end{array}\right)=1$, we get that if $r=0,4$, then $\wedge^{r} \rho_{A}$ is a character and thus the second part of the Tate conjecture is trivial in these cases. So the only interesting case is $r=2$. Then we have a 6 -dimensional Galois representation

$$
\wedge^{2} \rho_{A}: \Gamma_{F} \rightarrow \operatorname{Aut}\left(H_{e t}^{2}\left(A, \mathbb{Z}_{l}\right)\right) \simeq \mathrm{GL}_{6}\left(\mathbb{Z}_{l}\right) .
$$

But one can see easily (see for example Proposition 2.7 of $[\mathrm{G}]$ ) that

$$
\wedge^{2} \rho_{A} \simeq \xi_{l} \oplus \xi_{l} \omega_{K / F} \oplus \operatorname{Asai}_{K / F} \rho_{E},
$$

where $\xi_{l}$ is the $l$-adic cyclotomic character, $\omega_{K / F}$ is the quadratic character corresponding to $K / F$, and the Galois representation Asai $_{K / F} \rho_{E}$ of $\Gamma_{F}$ is the Asai representation associated to the Galois representation $\rho_{E}$ of $\Gamma_{K}$ relative to $K / F$. We know that Asai $_{K / F} \rho_{E}$ is a subrepresentation of

$$
\operatorname{Ind}_{\Gamma_{K}}^{\Gamma_{F}}\left(\rho_{E} \otimes \rho_{E}^{\tau}\right)
$$

which satisfies

$$
\left.\operatorname{Asai}_{K / F} \rho_{E}\right|_{\Gamma_{K}} \simeq \rho_{E} \otimes \rho_{E}^{\tau} \text {. }
$$


We know the following result (see Theorem A of [BGGT] and the remark after it; if $E$ has $\mathrm{CM}$, this result is trivial and the base change is arbitrary):

Theorem 4.1. With the same notations as above, if $F^{\prime}$ is a CM-extension of $F$, then there exists a CM-extension $K^{\prime \prime}$ of $K F^{\prime}$, which is Galois over $\mathbb{Q}$, such that $\left.\rho_{E}\right|_{\Gamma_{K^{\prime \prime}}}$ is automorphic, i.e. $\left.\rho_{E}\right|_{\Gamma_{K^{\prime \prime}}} \cong \rho_{\Pi^{\prime \prime}}$, where $\Pi^{\prime \prime}$ is an automorphic representation of $G L_{2}\left(\mathbb{A}_{K^{\prime \prime}}\right)$ and $\rho_{\Pi^{\prime \prime}}$ is an l-adic representation associated to $\Pi^{\prime \prime}$.

Let $F^{\prime}$ be a CM-field which contains $F$. From Theorem 4.1 we deduce that there exists a Galois CM-field $K^{\prime \prime}$ containing $K F^{\prime}$, such that $\left.\rho_{E}\right|_{\Gamma_{K^{\prime \prime}}} \cong \rho_{\Pi^{\prime \prime}}$, where $\Pi^{\prime \prime}$ is an automorphic representation of $\mathrm{GL}_{2}\left(\mathbb{A}_{K^{\prime \prime}}\right)$.

From Theorem 15.10 of [CR] we know that there exist some subfields $F_{i} \subseteq K^{\prime \prime}$, such that $F \subseteq F_{i}$ and $\operatorname{Gal}\left(K^{\prime \prime} / F_{i}\right)$ are solvable, and some integers $n_{i}$, such that the trivial representation

$$
1_{F^{\prime}}: \operatorname{Gal}\left(K^{\prime \prime} / F^{\prime}\right) \rightarrow \overline{\mathbb{Q}}^{\times}
$$

can be written as

$$
1_{F^{\prime}}=\sum_{i=1}^{u} n_{i} \operatorname{Ind}_{\operatorname{Gal}\left(K^{\prime \prime} / F_{i}\right)}^{\operatorname{Gal}\left(K^{\prime \prime} / F^{\prime}\right)} 1_{F_{i}} \quad \text { (a virtual sum) },
$$

from which we obtain

$$
L\left(s,\left.\operatorname{Asai}_{K / F} \rho_{E}\right|_{\Gamma_{F^{\prime}}}\right)=\prod_{i=1}^{u} L\left(s,\left.\operatorname{Asai}_{K / F} \rho_{E}\right|_{\Gamma_{F_{i}}}\right)^{n_{i}} .
$$

\subsection{Tate classes and poles of L-functions}

Assume that $F^{\prime}$ is a finite extension of $F$. Define:

$$
\mathbf{V}^{2}\left(A, F^{\prime}\right):=\left\{x \in H_{e t}^{2}\left(A, \overline{\mathbb{Q}}_{l}\right) \mid \wedge^{2} \rho_{A}(a) x=\xi_{l}(a) x, \text { for all } a \in \Gamma_{F^{\prime}}\right\},
$$

where $\xi_{l}$ is the $l$-adic cyclotomic character. The elements of $\mathbf{V}^{2}\left(A, F^{\prime}\right)$ are called Tate classes.

We will prove the following result:

Theorem 4.2. Assume that $F^{\prime}$ is a $C M$-field which contains $F$. Then the function $L\left(s,\left.\wedge^{2} \rho_{A}\right|_{\Gamma_{F^{\prime}}}\right)$ has a meromorphic continuation to the entire complex plane, and the order of the pole of $L\left(s,\left.\wedge^{2} \rho_{A}\right|_{\Gamma_{F^{\prime}}}\right)$ at $s=2$ is equal to $\operatorname{dim}_{\overline{\mathbb{Q}}_{l}} \mathbf{V}^{2}\left(A, F^{\prime}\right)$.

We consider

$$
\mathbf{V}^{2}\left(A, F_{i}\right):=\left\{x \in H_{e t}^{2}\left(A, \overline{\mathbb{Q}}_{l}\right) \mid \wedge^{2} \rho_{A}(a) x=\xi_{l}(a) x \text {, for all } a \in \Gamma_{F_{i}}\right\} .
$$


Since $1_{F^{\prime}}=\sum_{i=1}^{u} n_{i} \operatorname{Ind} \frac{\operatorname{Gal}\left(K^{\prime \prime} / F^{\prime}\right)}{\operatorname{Gal}\left(K^{\prime \prime} / F_{i}\right)} 1_{F_{i}}$, in order to prove Theorem 4.2 , it is sufficient to show the following result:

Proposition 4.3. For each $i$, the order of the pole of $L\left(s,\left.\wedge^{2} \rho_{A}\right|_{\Gamma_{F_{i}}}\right)$ at $s=2$ is equal to $\operatorname{dim}_{\overline{\mathbb{Q}}_{l}} \mathbf{V}^{2}\left(A, F_{i}\right)$.

\section{Proof.}

A) If $E$ is CM, we have that $\left.\wedge^{2} \rho_{A}\right|_{\Gamma_{F_{i}}}$ is a direct sum of monomial representations (see $\S 6.3$ of $[\mathrm{MR}]$ ). Here a monomial representation of $\Gamma_{F_{i}}$ is a representation which is induced from a one-dimensional representation of an open subgroup. Thus it is easy to see that the pole of $L\left(s,\left.\wedge^{2} \rho_{A}\right|_{\Gamma_{F_{i}}}\right)$ at $s=2$ is equal to the dimension of the space of Tate classes $\mathbf{V}^{2}\left(A, F_{i}\right)$ (so if $E$ is $\mathrm{CM}$, Theorem 4.2 is true actually for any $F^{\prime}$ ). Hence we are done if $E$ is CM.

B) If $E$ is non-CM, we distinguish two cases:

(i) Asai $\left._{K / F} \rho_{E}\right|_{\Gamma_{F_{i}}}$ is reducible. Then because Asai $\left._{K / F} \rho_{E}\right|_{\Gamma_{K}} \cong \rho_{E} \otimes \rho_{E}^{\tau}$, we get that the representation Asai $\left.\left.\left._{K / F} \rho_{E}\right|_{\Gamma_{K F_{i}}} \cong \rho_{E}\right|_{\Gamma_{K F_{i}}} \otimes \rho_{E}^{\tau}\right|_{\Gamma_{K F_{i}}}$, is reducible. Since the elliptic curve $E$ is non-CM, by applying Lemmas 3.1, 3.2 and 3.5, we deduce that $\rho_{E}^{\tau} \cong \rho_{E} \otimes \alpha$ for some Hecke character $\alpha$ of $K$. Hence, from Lemma 3.3, we know that $\alpha$ is a Hecke character of $I_{K}$ which is trivial on $I_{F}$. Therefore $\alpha$ can be written as $\alpha=\chi^{\tau} / \chi$ for some Hecke character $\chi$ of $I_{K}$. Hence

$$
\left(\rho_{E} \otimes \chi^{-1}\right)^{\tau} \cong \rho_{E} \otimes \chi^{-1} .
$$

So $\left.\rho_{E} \cong \rho_{E_{0}}\right|_{\Gamma_{K}} \otimes \chi$, where $\rho_{E_{0}}$ is some irreducible 2-dimensional nondihedral representation of $\Gamma_{F}$. Then from the properties of $\mathrm{Asai}_{K / F} \rho_{E}$ (see for example $[\mathrm{MP}]$ ), we have:

$$
\left.\operatorname{Asai}_{K / F} \rho_{E} \cong\left(\operatorname{Sym}^{2} \rho_{E_{0}} \oplus \omega_{E_{0}} \cdot \omega_{K / F}\right) \otimes \chi\right|_{I_{F}},
$$

where $\omega_{\pi_{0}}$ is the central character of $\rho_{E_{0}}$ and $\omega_{K / F}$ is the quadratic character corresponding to $K / F$.

Hence we obtain

$$
\left.\left.\left.\left.\left.\left.\operatorname{Asai}_{K / F} \rho_{E}\right|_{\Gamma_{F_{i}}} \cong \operatorname{Sym}^{2} \rho_{E_{0}}\right|_{\Gamma_{F_{i}}} \otimes \chi\right|_{I_{F}}\right|_{\Gamma_{F_{i}}} \oplus \omega_{E_{0}} \cdot \omega_{K / F} \cdot \chi\right|_{I_{F}}\right|_{\Gamma_{F_{i}}} .
$$

Since $\rho_{E_{0}}$ is non-dihedral, from Lemma 3.4 we deduce that $\left.\operatorname{Sym}^{2} \rho_{E_{0}}\right|_{\Gamma_{F_{i}}}$ is irreducible, and hence $\left.\mathrm{Asai}_{K / F} \rho_{E}\right|_{\Gamma_{F_{i}}}$ is a direct sum of an irreducible 3-dimensional representation and a character. Moreover since $\left.\rho_{E}\right|_{\Gamma_{K^{\prime \prime}}}$ is cuspidal automorphic ( $E$ is non-CM) and $\operatorname{Gal}\left(K^{\prime \prime} / F_{i}\right)$ is solvable, one can deduce easily from Langlands base change that $\left.\rho_{E_{0}}\right|_{\Gamma_{F_{i}}}$ is cuspidal automorphic (non-dihedral) and hence from Lemma 3.6 we get that $\left.\operatorname{Sym}^{2} \rho_{E_{0}}\right|_{\Gamma_{F_{i}}}$ is cuspidal automorphic. Thus the function $L\left(s,\left.\operatorname{Sym}^{2} \rho_{E_{0}}\right|_{\Gamma_{F_{i}}}\right)$ has no zeros or poles at $s=2$, and because of the irreducibility of $\left.\operatorname{Sym}^{2} \rho_{E_{0}}\right|_{\Gamma_{F_{i}}}$ one obtains trivially that Proposition 4.3 is true in this case. 
(ii) Asai $\left._{K / F} \rho_{E}\right|_{\Gamma_{F_{i}}}$ is irreducible. Then from Theorem D of [R2], and because $\left.\rho_{E}\right|_{\Gamma_{K^{\prime \prime}}}$ is cuspidal automorphic ( $E$ is non-CM) and $\operatorname{Gal}\left(K^{\prime \prime} / F_{i}\right)$ is solvable (and hence $\left.\rho_{E}\right|_{\Gamma_{K F_{i}}}$ is cuspidal non-dihedral), and using also part i) above, we get that Asai $\left.{ }_{K / F} \rho_{E}\right|_{\Gamma_{F_{i}}}$ is cuspidal automorphic. Thus the function $L\left(s\right.$, Asai $\left.\left._{K / F} \rho_{E}\right|_{\Gamma_{F_{i}}}\right)$ has no zeros or poles at $s=2$, and because of the irreducibility of $\left.\mathrm{Asai}_{K / F} \rho_{E}\right|_{\Gamma_{F_{i}}}$ one obtains trivially that Proposition 4.3 is true also in this case, and we are done.

\section{References}

[BGGT] T. Barnet-Lamb, T. Gee, D. Geraghty, R. Taylor, Potential automorphy and change of weight, Annals of Mathematics, to appear.

[CR] C.W. Curtis, I. Reiner, Methods of Representation Theory, Vol. I, Wiley, New York, 1981.

[G] G. van der Geer, Hilbert modular surfaces, Springer-Verlag 1988.

[GJ] S. Gelbart, H. Jacquet, A relation between automorphic representations of $G L(2)$ and $G L(3)$, Ann. Sci. École Norm. Sup. 11 (1979), 471-542.

[HLR] G. Harder, R.P. Langlands, M. Rapoport, Algebraische Zycklen auf Hilbert-Blumenthal-Flächen, J. Reine Angew. Math. 396 (1986), 53-120.

[K] A. Knightly, Tate classes on a Product of two Picard modular surfaces, J. Number Theory 107 (2004), 335-344.

[M] J.S. Milne, Abelian varieties, www.jmilne.org/math/CourseNotes/av.html

[MP] V.K. Murty, D. Prasad, Tate cycles on a product of two Hilbert modular surfaces, J. Number Theory 80(1) (2000), 25-43.

[MR] V.K. Murty, D. Ramakrishnan, Period relations and the Tate conjecture for Hilbert modular surfaces, Invent. Math. 89 (1987), 319-325.

[R1] D. Ramakrishnan, Modularity of the Rankin-Selberg L-series, and multiplicity one for SL(2), Ann. of Math. 152 (2000), 45-111.

[R2] D. Ramakrishnan, Modularity of solvable Artin representations of GO(4)-type, IMRN (2002), No. 1, 1-54.

[TA] J. Tate, Algebraic cycles and poles of zeta functions, In: Schilling, O.D.G. (ed.), Arithmetical algebraic geometry, New York: Harper and Row, 1966.

Address: Cristian Virdol: Department of Mathematics, Yonsei University, Seoul 120-749, Korea.

E-mail: virdol@yonsei.ac.kr

Received: 18 March 2013; revised: 31 March 2014 